\title{
Inclusive diffractive heavy quarkonium photoproduction including quark subprocesses
}

\author{
Jiayu Wu $\odot,{ }^{1, *}$ Yanbing Cai $\odot,{ }^{2, \dagger}$ and Wenchang Xiang ${ }^{2,1, 末}$ \\ ${ }^{1}$ Department of Physics, Guizhou University, Guiyang 550025, China \\ ${ }^{2}$ Guizhou Key Laboratory in Physics and Related Areas, Guizhou University of Finance and Economics, Guiyang 550025, China
}

(Received 29 November 2020; revised 16 June 2021; accepted 7 July 2021; published 28 July 2021; corrected 4 October 2021)

\begin{abstract}
To test the contributions of the quark subprocesses to the inclusive diffractive heavy quarkonium photoproduction, we first investigate the inclusive nondiffractive direct and resolved photoproduction of $J / \Psi$ in the framework of nonrelativistic quantum chromodynamics (NRQCD). We find that the theoretical total cross section of heavy quarkonium productions are in good agreement with the data available at HERA, once the $\gamma q, q g$, and $q q$ subprocesses in the heavy quark pair productions are taken into account. Then we predict the inclusive diffractive rapidity and transverse momentum distributions of $J / \Psi, \Psi(2 S)$, and $\Upsilon(1 S)$ in $p p, p \mathrm{~Pb}$, and $\mathrm{PbPb}$ collisions at the CERN Large Hadron Collider by using our quark improved NRQCD model. We find that the contributions from the quark involved subprocesses to quarkonium photoproduction are about $6 \%-15 \%$. The numerical results show that the contributions from quark involved subprocesses are significant modification in inclusive diffractive heavy quarkonium photoproduction.
\end{abstract}

DOI: 10.1103/PhysRevC.104.015204

\section{INTRODUCTION}

Since the discovery of the $J / \Psi$ charmonium in 1974 [1,2], the quarkonium production has attracted much attention experimentally and theoretically as it provides an excellent way to investigate both the perturbation and nonperturbation regimes of quantum chromodynamics. In recent years, a lot of experimental results about quarkonium production has been released. Such as the nuclear modification factor has been measured by the STAR Collaboration [3], PHENIX Collaboration [4], ALICE Collaboration [5], CMS Collaboration [6], ATLAS Collaboration [7], and LHCb Collaboration [8]. These results from different collision systems provide an unique opportunity to explore the mechanism of heavy quarkonium production. On the theoretical aspect, several approaches have been proposed to calculate the production of heavy quarkonium, such as the color singlet mechanism (CSM) [9-14], the color dipole mechanism [15-17], the colorevaporation model (CEM) [18-21], $k_{T}$-factorization method [22-24] and the NRQCD [25-27]. Among these approaches, the most widely used methods for heavy quarkonium production are CSM and NRQCD.

To describe the heavy quarkonium production, a key ingredient is the nonperturbative evolution of an intermediate

\footnotetext{
*wujyedu@gmail.com

†Corresponding author: myparticle@163.com

${ }^{\ddagger}$ wxiangphy@gmail.com
}

Published by the American Physical Society under the terms of the Creative Commons Attribution 4.0 International license. Further distribution of this work must maintain attribution to the author(s) and the published article's title, journal citation, and DOI. Funded by $S C O A P^{3}$.
$Q \bar{Q}$ pair into the final quarkonium. The CSM is the first model to describe this nonperturbative evolution. The CSM was successful in describing quarkonium production at low energy [28]. However, there are considerable discrepancies between CSM predictions and the experimental data at Tevatron. The CSM prediction of inclusive $J / \Psi$ hadroproduction underestimates the experimental data by more than one order of magnitude [29]. For inclusive $\Psi(2 S)$ hadroproduction, the discrepancy is even larger, reaching a factor of 50 [30]. The reason for these discrepancies is the coupled quantum number of the heavy quark pair is assumed to be in a color singlet state in the CSM. To solve this puzzle, Bodwin, Braaten, and Lepage proposed the NRQCD mechanism by introducing the color octet process [25]. In the NRQCD framework, the quarkonium can evolve from color singlet intermediate heavy quark pair, as well as color octet heavy quark pair, which greatly fill the gap between the CSM predictions and experimental measurements [31-33]. The theoretical results about quarkonium hadroproduction from NRQCD are also in good agreement with the experimental measurements of $\chi_{c J}$ and $\Upsilon(1 S)$ [34]. As NRQCD factorization method has achieved great success in description heavy quarkonium hadroproduction, many efforts have been made to investigate the heavy quarkonium photoproduction with NRQCD.

Photoproduction process is a vital process in electronproton deep inelastic scattering (DIS) and high energy ultraperipheral hadron-hadron collisions, since it offers significant information about the interaction mechanisms [35]. The $J / \Psi$ photoproduction at HERA has been studied in the framework of the NRQCD by taking into account the color octet contributions. It was found that the color octet matrix elements, which are obtained by fitting the hadroproduction data at the Tevatron, overestimate the HERA data an order of magnitude [36,37]. This excess is disappeared when the 
higher order effects are included [38]. The quarkonium photoproduction cross section at the CERN Large Hadron Collider (LHC) has been investigated by using the CSM in Ref. [39]. Meanwhile, the NRQCD has been used to study the inclusive nondiffractive and inclusive diffractive quarkonium photoproduction at LHC by combining with the resolved Pomeron model in Ref. [40]. Later on, the $\eta_{c}$ production has been performed in inclusive nondiffractive and diffractive processes with the NRQCD model in Ref. [41]. Although considerable efforts have been made to improve the performance of the theoretical models, the theoretical calculations can match with the experimental data under certain uncertainties, the mechanism for heavy quarkonium production is still far from the deep understanding. Therefore, a comprehensive analysis of the contributions from different subprocesses may be helpful for understanding the heavy quarkonium production mechanism.

In our previous study, the inclusive diffractive heavy quarkonium photoproduction has been investigated by taking into account the $g+g \rightarrow Q \bar{Q}+g$ subprocess where one of the initial gluons comes from the resolved photon and the other comes from resolved Pomeron [42]. We found that the resolved photoproduction process has significant contributions to the heavy quarkonium production. However gluons are not the only constituents in the hadron-like photons and hadron-like Pomerons. In this paper, we take into account the quarks constituents to investigate the inclusive diffractive heavy quarkonium photoproduction on top of our previous work [42]. We find that the contributions from the quark involved subprocesses to quarkonium photoproduction are about 6\%-15\%, which indicates that quark-initiated channels have significant modification to the quarkonium production.

\section{INCLUSIVE DIFFRACTIVE QUARKONIUM PHOTOPRODUCTION IN NRQCD}

In this section, we first introduce the mechanism of inclusive diffractive heavy quarkonium photoproduction based on the NRQCD combined with the resolved Pomeron model. Then we give the total cross section for inclusive diffractive heavy quarkonium production in direct and resolved photoproduction processes. The total cross section and the rapidity distribution of the inclusive diffractive heavy quarkonium photoproduction in $p p, p \mathrm{~Pb}$, and $\mathrm{PbPb}$ collisions are presented at the end of this section.

\section{A. The mechanism of inclusive diffractive quarkonium photoproduction}

The NRQCD is one of the most widely used theoretical tool to describe the quarkonium production. In the NRQCD formalism, the cross section for the production of a heavy quarkonium $H$ can be factorized as [25]

$$
\sigma(a b \rightarrow H+X)=\sum_{n} \sigma(a b \rightarrow Q \bar{Q}[n]+X)\left\langle\mathcal{O}_{[n]}^{H}\right\rangle
$$

where $\sigma(a b \rightarrow Q \bar{Q}[n]+X)$ is the short distance process and $\left\langle O_{[n]}^{H}\right\rangle$ is the long distance process. In the short distance process, a pair of quark-antiquark is produced at collision point, and the quark-antiquark forms a special system $Q \bar{Q}[n]$ which can be a color singlet state or color octet state. In the long distance process, the special system form a bound state, and eventually the bound state evolutes into the final quarkonium through soft gluon emission. In the NRQCD formalism, the short distance matrix elements can be calculated perturbatively, but the long distance matrix is a nonperturbative process and it is usually given by the lattice QCD calculation or extracting from experimental data.

In this work, we combine the NRQCD factorization approach with resolved Pomeron model to investigate the inclusive diffractive heavy quarkonium photoproduction in $p p, p \mathrm{~Pb}$, and $\mathrm{PbPb}$ collisions. To better illustrate the inclusive diffractive processes, we introduce some related conceptions. The inclusive nondiffractive events are defined that the final particle is produced by hadron breakup on one side. The diffractive events are refer to the fact the hadrons are intact after the interaction. In the resolved Pomeron model, the inclusive diffractive events mean that the final particle is produced with the remnants of the Pomerons while the hadrons are intact. As the photoproduction has direct and resolved processes according to the scattering mechanism of the photon, we shall consider inclusive diffractive direct photoproduction process and inclusive diffractive resolved photoproduction process as shown in Fig. 1. For simplicity, we use $p p$ collisions as an example to introduce these two kinds of inclusive diffractive processes. The left panel of Fig. 1 shows the inclusive diffractive direct photoproduction process. In this process, the photons emitted by the proton $(A)$ interact with the partons (quarks and gluons) from the resolved Pomerons in the proton $(B)$ to produce the final heavy quarkonium $H$. The right panel of Fig. 1 shows the inclusive diffractive resolved photoproduction process. In this process, due to the Heisenberg uncertainty principle, the energetic photons from the energetic proton $(A)$ can fluctuate into partons (quarks and gluons). Then these partons interact with the partons from the resolved Pomerons to form the final heavy quarkonium $H$. For inclusive diffractive direct photoproduction process and inclusive diffractive resolved photoproduction process, the heavy quarkonium $H$ is produced with the remnants of the Pomerons and photons (in the resolved photon case) while the two incident protons are intact. Therefore, both processes mentioned above belong to inclusive diffractive photoproduction.

\section{B. The cross section of the inclusive diffractive direct photoproduction}

For the inclusive diffractive direct photoproduction process, the total cross section can be factorized into the partonic distribution function and differential cross section

$$
\begin{aligned}
& \sigma_{\mathrm{dir}}(\gamma+B \rightarrow H+X) \\
& \quad=\int d z d p_{T}^{2} \frac{x_{\mathrm{dir}} f_{b / B}\left(x_{\mathrm{dir}}, Q^{2}\right)}{z(1-z)} \frac{d \sigma}{d t}(\gamma+b \rightarrow H+X),
\end{aligned}
$$

where $f_{b / B}\left(x_{\mathrm{dir}}, Q^{2}\right)$ is the diffractive parton distribution and $z$ is the fraction of the photon energy carried by the quarkonium. 

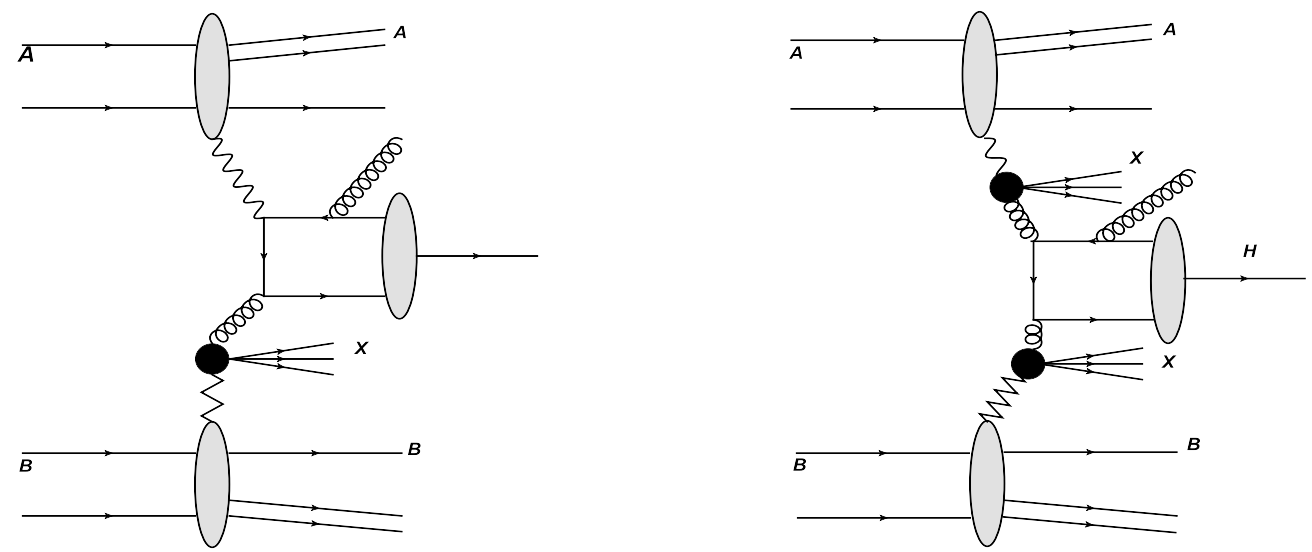

FIG. 1. The schematic diagrams of inclusive diffractive heavy quarkonium direct (left panel) and resolved (right panel) photoproduction in the resolved Pomeron model.

The $x_{\text {dir }}$ is the momentum fraction of proton carried by the quark or gluon and can be written as

$$
x_{\mathrm{dir}}=\frac{p_{T}^{2}+M^{2}(1-z)}{W_{\gamma p}^{2} z(1-z)},
$$

where $p_{T}$ is the transverse momentum of the quarkonium and $M$ is the mass of the quarkonium. The $W_{\gamma p}$ in Eq. (3) is the $\gamma p$ center-of-mass energy

$$
W_{\gamma p}=\sqrt{2 \omega \sqrt{s}}
$$

where $\sqrt{s}$ represents the center-of-mass energy of the $p p$ collision system and $\omega$ is the photon energy

$$
\omega=\frac{M}{2} \exp ( \pm y) \text {. }
$$

It should be noted that formalism in Eq. (2) seems to be the same as the inclusive nondiffractive quarkonium photoproduction process at first sight. Indeed, the inclusive diffractive direct photoproduction process is quite different from the inclusive nondiffractive quarkonium photoproduction process because $f_{b / B}\left(x_{\mathrm{dir}}, Q^{2}\right)$ in Eq. (2) is the diffractive parton distribution rather than the inclusive parton distribution. The diffractive parton distribution of a proton can be expressed as the convolution of the Pomeron flux in the proton and parton distribution in the Pomeron [43]

$$
f_{b / p}\left(x, Q^{2}\right)=\int_{x}^{1} \frac{d x_{\mathcal{P}}}{x_{\mathcal{P}}} f_{\mathcal{P}}^{p}\left(x_{\mathcal{P}}\right) g_{\mathcal{P}}\left(\frac{x}{x_{\mathcal{P}}}, Q^{2}\right),
$$

where the Pomeron fluxes emitted by the proton is given by

$$
f_{\mathcal{P}}^{p}\left(x_{\mathcal{P}}\right)=\int_{t_{\min }}^{t_{\max }} d t f_{\mathcal{P}}^{p}\left(x_{\mathcal{P}}, t\right)=\int_{t_{\min }}^{t_{\max }} \frac{\lambda e^{\beta t}}{x_{\mathcal{P}}^{2 \alpha_{\mathcal{P}}(t)-1}} d t
$$

with

$$
\begin{aligned}
t_{\max } & =\frac{-m_{p}^{2} x_{\mathcal{P}}^{2}}{1-x_{\mathcal{P}}}, \\
t_{\min } & =-1 \mathrm{GeV}^{2} .
\end{aligned}
$$

In Eq. (6), $g_{\mathcal{P}}\left(\frac{x}{x_{\mathcal{P}}}, Q^{2}\right)$ is the parton distribution in Pomeron, which is given by a parametrized formula in Ref. [44].
In this study, we will give predictions of inclusive diffractive heavy quarkonium photoproduction in $\mathrm{pPb}$ and $\mathrm{PbPb}$ collisions. Thus, we introduce the diffractive parton distribution of a lead by following Ref. [45], which can be expressed as

$$
\begin{aligned}
f_{b / P b}\left(x_{\mathcal{P}}\right) & =\int_{t_{\min }}^{t_{\max }} d t f_{\mathcal{P}}^{\mathrm{Pb}}\left(x_{\mathcal{P}}, t\right) \\
& =R_{g} A^{2} \int_{t_{\min }}^{t_{\max }} \frac{\lambda e^{\beta t}}{x_{\mathcal{P}}^{2 \alpha \mathcal{P}(t)-1}} d t F_{A}^{2}(t),
\end{aligned}
$$

where $R_{g}$ is the suppression factor associated to the nuclear shadowing and $F_{A}(t)$ is the nuclear form factor.

The distributions in Eqs. (6) and (10) are key ingredients to calculate the inclusive diffractive quarkonium photoproduction. We would like to note that the diffractive parton distribution, Eq. (6), was employed to compute the inclusive diffractive direct photoproduction of $J / \Psi, \Psi(2 S)$, and $\Upsilon(1 S)$ [40]. However, the quark contributions were not considered in Ref. [40]. In this work, we take into account the quark processes to investigate the inclusive diffractive heavy quarkonium photoproduction by combining the NRQCD model with resolved Pomeron model. We call this approach as quark improved NRQCD model. The quarks have two resources, one of the sources of quarks is the hadron-like Pomerons. So, we include quarks emitted from hadron-like Pomerons on top of the gluon subprocesses

$$
\begin{aligned}
& \gamma+g \rightarrow Q \bar{Q}\left[{ }^{3} S_{1}^{(1)},{ }^{3} S_{0}^{(8)},{ }^{3} S_{1}^{(8)},{ }^{3} P_{0,1,2}^{(8)}\right]+g, \\
& \gamma+q \rightarrow Q \bar{Q}\left[{ }^{3} S_{0}^{(8)},{ }^{3} S_{1}^{(8)},{ }^{3} P_{0,1,2}^{(8)}\right]+q .
\end{aligned}
$$

The other sources of quarks is the hadron-like photons, which shall be introduced in the following subsection.

\section{The cross section of the inclusive diffractive resolved photoproduction}

According to the Heisenberg uncertainty principle, the energetic photon emitted from the projectile hadron can be treated as hadron-like photon which can fluctuate into quarks and gluons. Then the quarks or gluons interact with the partons inside a Pomeron which is emitted from the target hadron. The two incident hadrons remain intact. We usually 
call this process as the inclusive diffractive resolved photoproduction process. According to the factorization formalism, the cross section for the inclusive diffractive resolved photoproduction process can be written as

$$
\begin{aligned}
& \sigma_{\text {res }}(\gamma+B \rightarrow H+X) \\
& =\int d z d p_{T}^{2} d x \frac{x_{\text {res }} f_{a / \gamma}\left(x_{\text {res }}, Q^{2}\right) f_{b / B}\left(x, Q^{2}\right)}{z\left(1-\frac{z}{x}\right)} \frac{d \sigma}{d t} \\
& \quad \times(a+b \rightarrow H+X),
\end{aligned}
$$

where $f_{a / \gamma}\left(x_{\text {res }}, Q^{2}\right)$ is the parton distribution function of the hadron-like photon [46], and $x_{\text {res }}$ is the momentum fraction of photon carried by the quark or gluon

$$
x_{\mathrm{res}}=\frac{x p_{T}^{2}+M^{2}(x-z)}{W_{\gamma p}^{2} x z\left(1-\frac{z}{x}\right)} .
$$

In inclusive diffractive resolved photoproduction process, both the photon and the Pomeron are the hadron-like objects. So the parton $a$ and the parton $b$ in Eq. (13) can either come from the hadron-like photon or come from the hadron-like Pomeron. To improve the calculation accuracy, one should take into account the contributions both from the gluon subprocesses and quark subprocesses. Therefore, for inclusive diffractive resolved photoproduction process in our quark improved NRQCD model, we have the following subprocesses:

$$
\begin{aligned}
& g+g \rightarrow Q \bar{Q}\left[{ }^{3} S_{1}^{(1)},{ }^{3} S_{0}^{(8)},{ }^{3} S_{1}^{(8)},{ }^{3} P_{0,1,2}^{(8)}\right]+g, \\
& g+q \rightarrow Q \bar{Q}\left[{ }^{3} S_{0}^{(8)},{ }^{3} S_{1}^{(8)},{ }^{3} P_{0,1,2}^{(8)}\right]+q, \\
& q+\bar{q} \rightarrow Q \bar{Q}\left[{ }^{3} S_{0}^{(8)},{ }^{3} S_{1}^{(8)},{ }^{3} P_{0,1,2}^{(8)}\right]+g .
\end{aligned}
$$

Using Eqs. (15)-(17), we can calculate the heavy quarkonium photoproduction from inclusive diffractive resolved photoproduction process. Together with the contributions from inclusive diffractive direct photoproduction process calculated by using Eqs. (11) and (12), we can obtain the inclusive diffractive photoproduction cross section. In addition, using Eqs. (12), (16), and (17), we can estimate the contributions from quark subprocesses.

\section{The total cross section and the rapidity distribution of the inclusive diffractive photoproduction}

In this subsection, we will first discuss the total cross section of the inclusive diffractive heavy quarkonium photoproduction which includes the contributions from the inclusive diffractive direct photoproduction process and the inclusive diffractive resolved photoproduction process. Then we give the rapidity distribution on the basis of the total cross section. The total cross section for the heavy quarkonium in the inclusive diffractive photoproduction is given by

$$
\begin{aligned}
& \sigma_{\mathrm{tot}}(A+B \rightarrow A \otimes H+X \otimes B) \\
& =\int d \omega \frac{d N_{\gamma / A}(\omega)}{d \omega} \sigma_{\gamma B} \rightarrow H X \otimes B \\
& \quad+\int d \omega \frac{d N_{\gamma / B}(\omega)}{d \omega} \sigma_{\gamma A} \rightarrow H X \otimes A,
\end{aligned}
$$

where $\otimes$ denotes the existence of a rapidity gap in the final state and $\omega$ denotes the energy of photon. The cross section $\sigma_{\gamma B} \rightarrow H X \otimes B$ denotes the inclusive diffractive photoproduction cross section and can be obtained from the sum of the inclusive diffractive direct photoproduction process Eq. (2) and the inclusive diffractive resolved photoproduction process Eq. (13). A similar definition can be established for $\sigma_{\gamma A} \rightarrow$ $H X \otimes A$. The $A$ and $B$ denote the hadron or nucleus. For $p p$ collisions, using the relationship between rapidity and energy in Eq. (5), the rapidity distribution can be written as

$$
\begin{aligned}
\frac{d \sigma_{\mathrm{tot}}^{p p \rightarrow p H+X p}}{d y}= & {\left[\omega \frac{d N_{\gamma}^{p}}{d \omega} \sigma^{\gamma p \rightarrow H X p}\right]_{\omega=\omega_{l}} } \\
& +\left[\omega \frac{d N_{\gamma}^{p}}{d \omega} \sigma^{\gamma p \rightarrow H X p}\right]_{\omega=\omega_{r}},
\end{aligned}
$$

where the subscripts $l(r)$ denote photon flux from the left (right) proton. The equivalent photon flux $d N_{\gamma}^{p} / d \omega$ of the relativistic proton in Eq. (19) is given by [47]

$$
\begin{aligned}
\frac{d N_{\gamma}^{p}}{d \omega}= & \frac{\alpha_{e m}}{2 \pi \omega}\left[1+\left(1-\frac{2 \omega}{\sqrt{s}}\right)^{2}\right] \\
& \times\left(\ln \Omega-\frac{11}{6}+\frac{3}{\Omega}-\frac{3}{2 \Omega^{2}}+\frac{1}{3 \Omega^{3}}\right)
\end{aligned}
$$

with

$$
\Omega=1+\left[\left(0.71 \mathrm{GeV}^{2}\right) / Q_{\min }^{2}\right],
$$

where $Q_{\min }^{2}=\omega^{2} /\left[\gamma_{L}^{2}(1-2 \omega / \sqrt{s})\right] \approx\left(\omega / \gamma_{L}\right)^{2}$ and $\gamma_{L}$ is the Lorentz factor.

For $p \mathrm{~Pb}$ collisions, the rapidity distribution is

$$
\begin{aligned}
\frac{d \sigma_{\mathrm{tot}}^{p \mathrm{~Pb} \rightarrow p H X \mathrm{~Pb}}}{d y}= & {\left[\omega \frac{d N_{\gamma}^{\mathrm{Pb}}}{d \omega} \sigma^{\gamma P b \rightarrow H X p}\right]_{\omega=\omega_{l}} } \\
& +\left[\omega \frac{d N_{\gamma}^{p}}{d \omega} \sigma^{\gamma p \rightarrow H X \mathrm{~Pb}}\right]_{\omega=\omega_{r}},
\end{aligned}
$$

where $d N_{\gamma}^{\mathrm{Pb}} / d \omega$ denotes the photon flux of the lead [48]

$$
\frac{d N_{\gamma}^{\mathrm{Pb}}}{d \omega}=\frac{2 Z^{2} \alpha_{e m}}{\pi \omega}\left[\xi K_{0}(\xi) K_{1}(\xi)-\frac{\xi^{2}}{2}\left(K_{1}^{2}(\xi)-K_{0}^{2}(\xi)\right)\right]
$$

with $\xi=\omega\left(R_{p}+R_{\mathrm{Pb}}\right) / \gamma_{L}$. The $K_{0}$ and $K_{1}$ are the modified Bessel functions and $Z$ is atomic number. Since the photon flux of the nuclei is larger than the photon flux of the proton, the rapidity distribution should be obvious asymmetry with a great peak at the lead side in the $p \mathrm{~Pb}$ collisions.

In addition to the rapidity distribution of heavy quarkonium production in $p p$ and $p \mathrm{~Pb}$ collisions, we also study the heavy quarkonium photoproduction in the $\mathrm{PbPb}$ collisions by including the quark involved subprocesses as mentioned above. The rapidity distribution of the $\mathrm{PbPb}$ collisions can be written as

$$
\begin{aligned}
\frac{d \sigma_{\mathrm{tot}}^{\mathrm{Pb} \rightarrow \mathrm{Pb} H X \mathrm{~Pb}}}{d y}= & {\left[\omega \frac{d N_{\gamma}^{\mathrm{Pb}}}{d \omega} \sigma^{\gamma \mathrm{Pb} \rightarrow H X \mathrm{~Pb}}\right]_{\omega=\omega_{l}} } \\
& +\left[\omega \frac{d N_{\gamma}^{\mathrm{Pb}}}{d \omega} \sigma^{\gamma \mathrm{Pb} \rightarrow H X \mathrm{~Pb}}\right]_{\omega=\omega_{r}} .
\end{aligned}
$$


TABLE I. Values of long-distance matrix elements for $J / \Psi, \Psi(2 S)$, and $\Upsilon(1 S)$.

\begin{tabular}{lccc}
\hline \hline$\left(\mathrm{GeV}^{3}\right)$ & $J / \Psi$ & $\Psi(2 S)$ & $\Upsilon(1 S)$ \\
\hline$\left\langle\mathcal{O}\left[{ }^{3} S_{1}^{[1]}\right]\right\rangle$ & 1.2 & 0.76 & 10.9 \\
$\left\langle\mathcal{O}\left[{ }^{1} S_{0}^{[8]}\right]\right\rangle$ & $0.018 \pm 0.0087$ & $0.0080 \pm 0.0067$ & $0.0121 \pm 0.00400$ \\
$\left\langle\mathcal{O}\left[{ }^{3} S_{1}^{[8]}\right]\right\rangle$ & $0.0013 \pm 0.0013$ & $0.00330 \pm 0.0021$ & $0.0477 \pm 0.0334$ \\
$\left\langle\mathcal{O}\left[{ }^{3} P_{0}^{[8]}\right]\right\rangle$ & $(0.018 \pm 0.0087) m_{c}^{2}$ & $3 \times(0.0080 \pm 0.0067) m_{c}^{2}$ & $5 \times(0.121 \pm 0.00400) m_{b}^{2}$ \\
$\left\langle\mathcal{O}\left[{ }^{3} P_{1}^{[8]}\right]\right\rangle$ & $3 \times(0.018 \pm 0.0087) m_{c}^{2}$ & $5 \times(0.0080 \pm 0.0067) m_{c}^{2}$ & - \\
$\left\langle\mathcal{O}\left[{ }^{3} P_{2}^{[8]}\right]\right\rangle$ & $5 \times(0.018 \pm 0.0087) m_{c}^{2}$ & - \\
\hline \hline
\end{tabular}

Using the formalisms introduced in this section, together with the partonic subprocesses introduced in the last two subsections, we can estimate the observations of the inclusive diffractive heavy quarkonium photoproduction.

\section{RESULTS}

In this section, we shall present the heavy photoproduction results with our quark improved NRQCD model. To test the contributions of the quark subprocesses to the inclusive diffractive heavy quarkonium photoproduction, we first investigate the inclusive nondiffractive $J / \Psi$ photoproduction in $p p$ collisions and compare our theoretical calculations with the $J / \Psi$ experimental data from $H 1$ Collaboration [49]. Then we use the quark improved NRQCD model to make predictions for the rapidity and transverse momentum distributions of the inclusive diffractive $J / \Psi, \Psi(2 S)$, and $\Upsilon(1 S)$ photoproduction in $p p, p \mathrm{~Pb}$, and $\mathrm{PbPb}$ collisions. We would like to point out that the parton distribution used in the calculations of inclusive nondiffractive photoproduction and the inclusive diffractive photoproduction are inclusive parton distribution [50] and inclusive diffractive parton distribution [44], respectively.

In our studies, the masses of charm quark and bottom quark are taken $1.5 \mathrm{GeV}$ and $4.5 \mathrm{GeV}$, respectively. The fraction of photon energy carried by the quarkonium is integrated over the range $0.3 \leqslant z \leqslant 0.9$ as the experimental measurement is performed in this range [49]. The minimum value of the transverse momentum is set to $1 \mathrm{GeV}$. We set the factorization scale as $Q^{2}=p_{T}^{2}$. In addition, the differential cross section of the partonic subprocesses is calculated in Ref. [51], and the long-distance matrix elements are listed in Table I [52].

To test the contributions of the quark subprocesses to the inclusive diffractive heavy quarkonium photoproduction, we first present the energy dependence of the inclusive nondiffractive $J / \Psi$ total cross section in $p p$ collisions as shown in Fig. 2. The theoretical results are calculated by Eqs. (2) and (13) with inclusive parton distribution of the proton and the experimental data are taken from the H1 Collaboration [49]. The dashed purple line is the contributions from the $\gamma g$ direct photoproduction process. The dotted green line is the results from the $g g$ resolved photoproduction process. The dot-dashed orange line is the contributions from the processes of quarks $(\gamma q, q q, q g)$, which are introduced, for the first time, into theoretical calculations of the heavy quarkonium photoproduction in the resolved Pomeron framework. The solid red line is the total cross section. The bands are the uncertainties from factorization scale choices $\left(Q^{2}=p_{T}^{2} / 2\right.$ and $\left.2 p_{T}^{2}\right)$ and the long-distance matrix elements (similarly hereinafter). In our plots, we show the uncertainties for both the sum of all channels and the quark involved channel. As one can see that the quark improved NRQCD model can give a rather successful description of the $J / \Psi$ photoproduction data from the H1 Collaboration, which indicates that the quark involved three subprocesses have significant contributions.

In this study, we focus on the photoproduction of $J / \Psi$, $\Psi(2 S)$, and $\Upsilon(1 S)$. For completeness, in Fig. 3, we also give predictions for the inclusive nondiffractive total cross section of $\Psi(2 S)$ and $\Upsilon(1 S)$ with the same strategy used in Fig. 2. We can see that the quark involved three subprocesses also have significant contributions to the $\Psi(2 S)$ and $\Upsilon(1 S)$ productions. From Figs. 2 and 3, we can see that in the low region of center of mass (or large $x$ ), the contributions of the quark involved three subprocesses are larger than the ones from $g g$ processes, since the quark distribution is larger than the gluon distribution function in the low energy region.

From the inclusive nondiffractive heavy quarkonium photoproduction at HERA energies, one can see that the quark involved three subprocesses have significant contributions to the total cross section. It is reasonable to believe that these three subprocesses mentioned above are also important in the inclusive diffractive heavy quarkonium production at LHC energies. So, we use the quark improved NRQCD model to study the inclusive diffractive heavy quarkonium photoproduction at LHC energies through using diffractive parton distribution. Using Eq. (19) with diffractive parton distribution in Eq. (6), we calculate the predictions of the rapidity distribution for the inclusive diffractive $J / \Psi, \Psi(2 S)$, and $\Upsilon(1 S)$ in $p p$ collisions

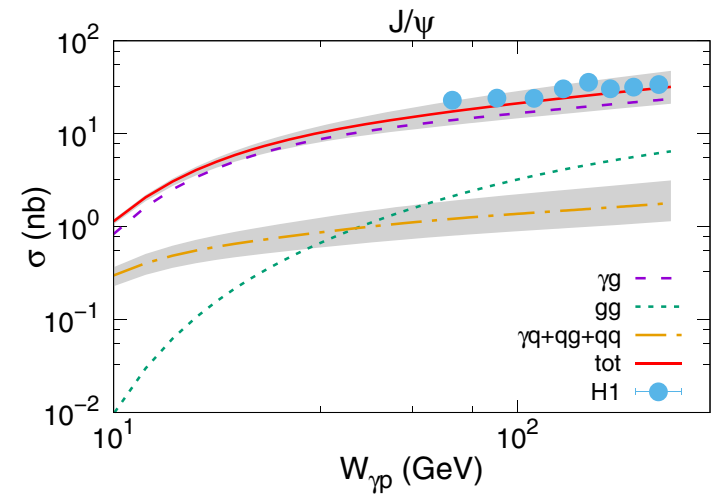

FIG. 2. The energy dependence of the cross section for the $J / \Psi$ photoproduction in inclusive nondiffractive $\gamma p$ interactions. Data are from the H1 Collaboration [49]. 

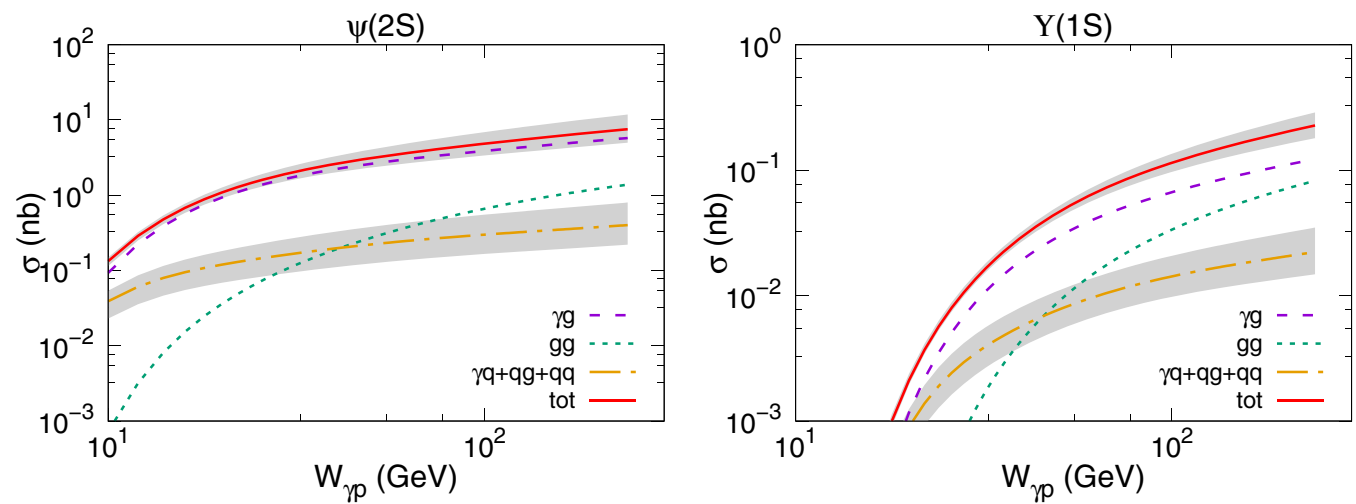

FIG. 3. Predictions for the energy dependence of the cross section for the $\Psi(2 S)$ and $\Upsilon(1 S)$ photoproduction in inclusive nondiffractive $\gamma p$ interaction.

at $\sqrt{s}=5.02 \mathrm{TeV}$, the relevant numerical results are shown in Fig. 4.

In the LHC energies, $p \mathrm{~Pb}$ and $\mathrm{PbPb}$ collisions have also attracted much attention. So we calculate the predictions of rapidity distributions for these two collision processes via Eqs. (22) and (24) with diffractive parton distribution in Eqs. (6) and (10). We present the numerical calculations of the rapidity distributions for the inclusive diffractive $J / \Psi, \Psi(2 S)$, and $\Upsilon(1 S)$ photoproduction in $p \mathrm{~Pb}$ collisions at $\sqrt{s}=5.02$ $\mathrm{TeV}$ in Fig. 5. As expected, in $p \mathrm{~Pb}$ collisions the rapidity distributions are asymmetric, with the peak at the lead side, since the photon spectrum of a nucleus is much larger than a proton.

The rapidity distributions for the inclusive diffractive $J / \Psi$, $\Psi(2 S)$, and $\Upsilon(1 S)$ photoproduction in $\mathrm{PbPb}$ collisions at $\sqrt{s}=5.02 \mathrm{TeV}$ are shown in Fig. 6 . As in the $p p$ collisions, the total rapidity distributions are symmetric about the midrapidity. However, the rapidity distributions are larger than the $p p$ collisions due to the enhancement of the photon flux.

From Figs. 2-6, we can see that the relative contribution of the $g g$ channel to the $\Upsilon(1 S)$ production is larger than the relative contribution of the same channel to the charmonium $[J / \Psi$ and $\Psi(2 S)]$ production. It is because of the different squared charge factor of quarks $Q_{q}^{2}\left(Q_{c}^{2}=4 / 9\right.$ and $\left.Q_{b}^{2}=1 / 9\right)$ in the differential cross section. According to Ref. [51], there is not $Q_{q}^{2}$ in the differential cross section for $g g$ channel. Therefore, there is not suppression in the differential cross section in $g g$ channel for both bottomonium and charmonium production in terms of the squared charge factor. However, the differential cross section for $\gamma g$ channel is proportional to $Q_{q}^{2}$. Consequently, the differential cross section in the $\gamma g$ channel for bottomonium is suppressed by a factor of four as compared with the one for charmonium in terms of the squared charge factor. Taking Fig. 4 as an example, we can see that the dominant contribution to the total differential cross section are from $\gamma g$ channel and $g g$ channel. As a consequence, the contribution of the $g g$ channel to bottomonium production is relatively larger than the contribution of the $g g$ channel to charmonium production.

In Figs. 7-9, we present the predictions of the transverse momentum distribution for the inclusive diffractive $J / \Psi$, $\Psi(2 S)$, and $\Upsilon(1 S)$ photoproduction at central rapidities $(y=$ 0 ) in $p p, p \mathrm{~Pb}$, and $\mathrm{PbPb}$ collisions at $\sqrt{s}=5.02 \mathrm{TeV}$. As expected that the transverse momentum distributions of $\Upsilon(1 S)$ are smaller than that of $J / \Psi$ and $\Psi(2 S)$, due to the fact that the mass of $\Upsilon(1 S)$ is larger than charmonium.

We would like to point out that an accurate theoretical prediction in NRQCD is hampered by large uncertainties. From the bands in the above figures, we can see that there are relatively large uncertainties stemming from factorization scale choices and the long-distance matrix elements. It is understandable since there are large uncertainties that arise from the long-distance matrix elements (see Table I). We estimate the percentage of the contributions from quark-initiated channels and the uncertainties of the total differential cross section. It find that they are about $6 \%-15 \%$ and $10 \%-25 \%$, respectively.
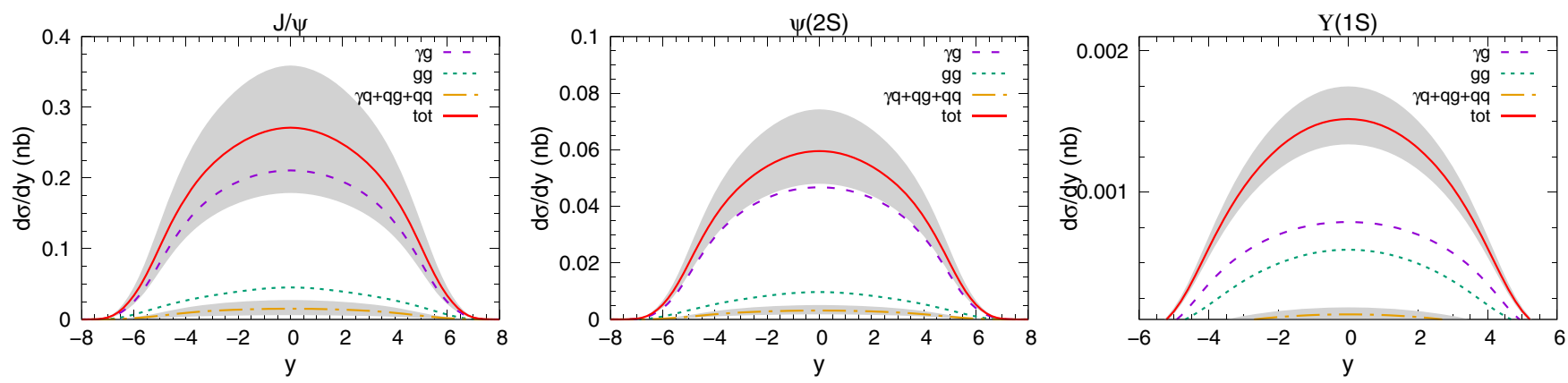

FIG. 4. Rapidity distributions for the inclusive diffractive $J / \Psi, \Psi(2 S)$, and $\Upsilon(1 S)$ photoproduction in $p p$ collisions at $\sqrt{s}=5.02 \mathrm{TeV}$. 

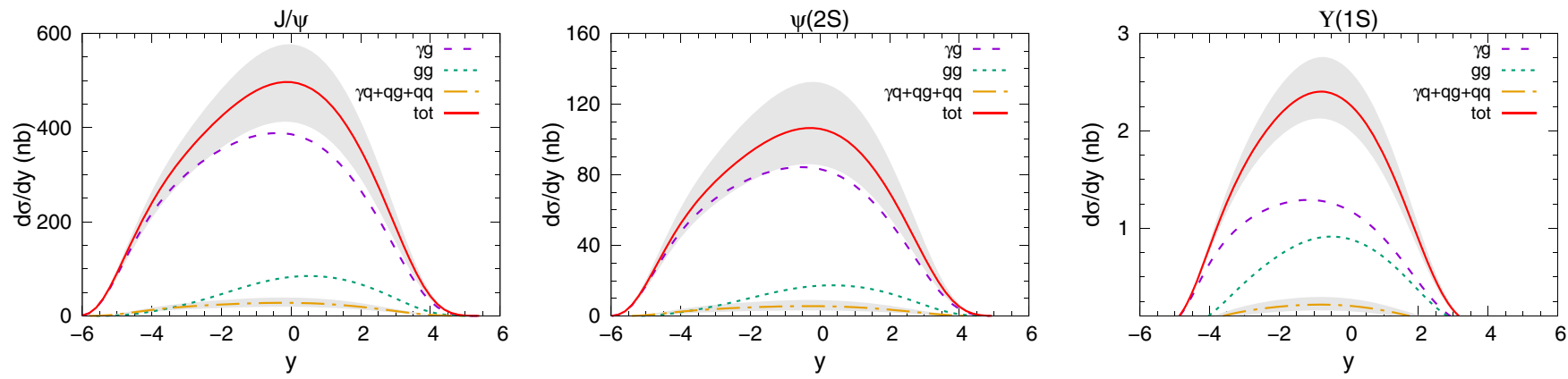

FIG. 5. Rapidity distributions for the inclusive diffractive $J / \Psi, \Psi(2 S)$, and $\Upsilon(1 S)$ photoproduction in $p \mathrm{~Pb}$ collisions at $\sqrt{s}=5.02 \mathrm{TeV}$.
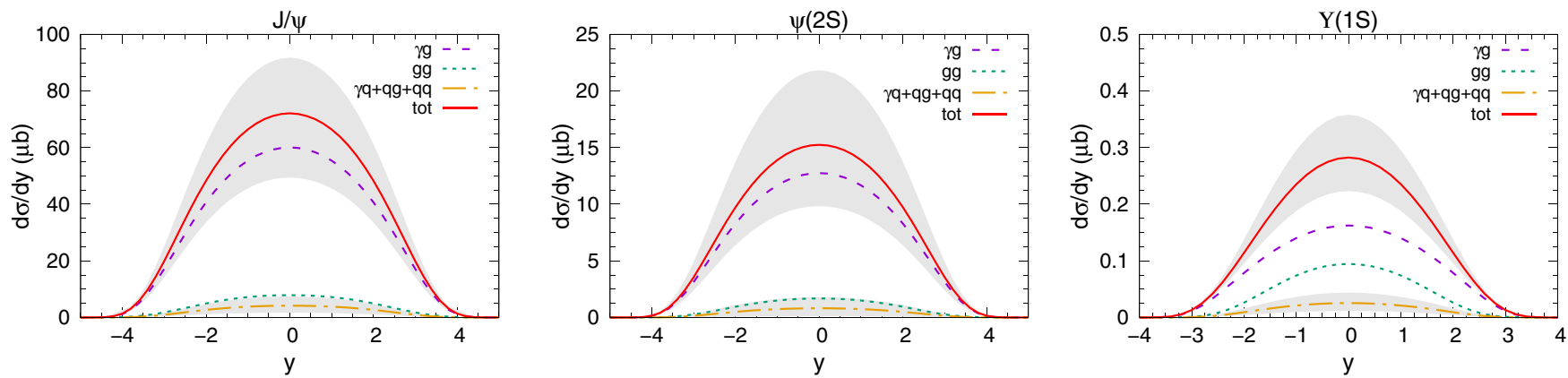

FIG. 6. Rapidity distributions for the inclusive diffractive $J / \Psi, \Psi(2 S)$, and $\Upsilon(1 S)$ photoproduction in $\mathrm{PbPb}$ collisions at $\sqrt{s}=5.02 \mathrm{TeV}$.
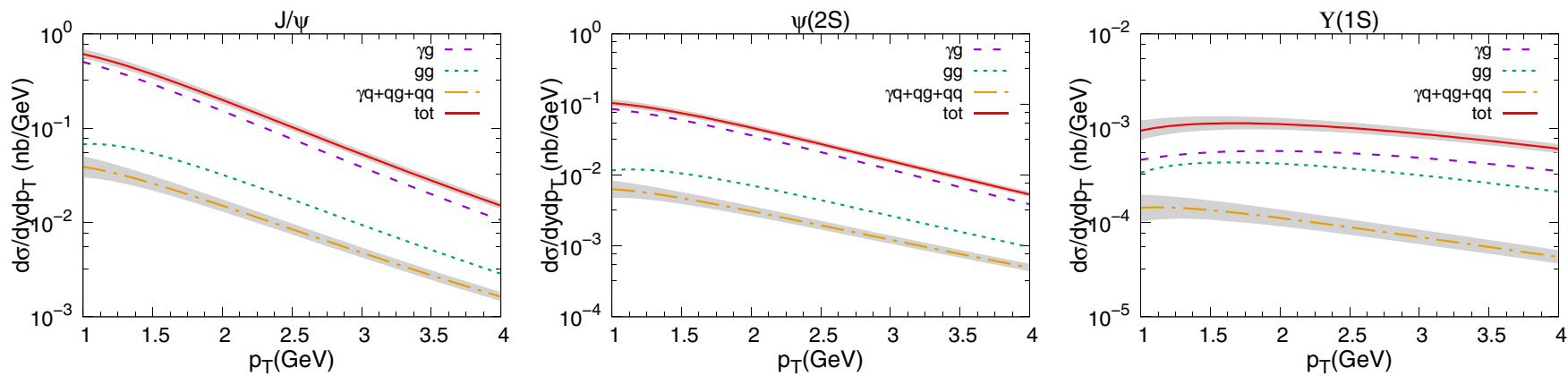

FIG. 7. Transverse momentum distributions for the inclusive diffractive $J / \Psi, \Psi(2 S)$, and $\Upsilon(1 S)$ photoproduction at central rapidities $(y=0)$ in $p p$ collisions at $\sqrt{s}=5.02 \mathrm{TeV}$.
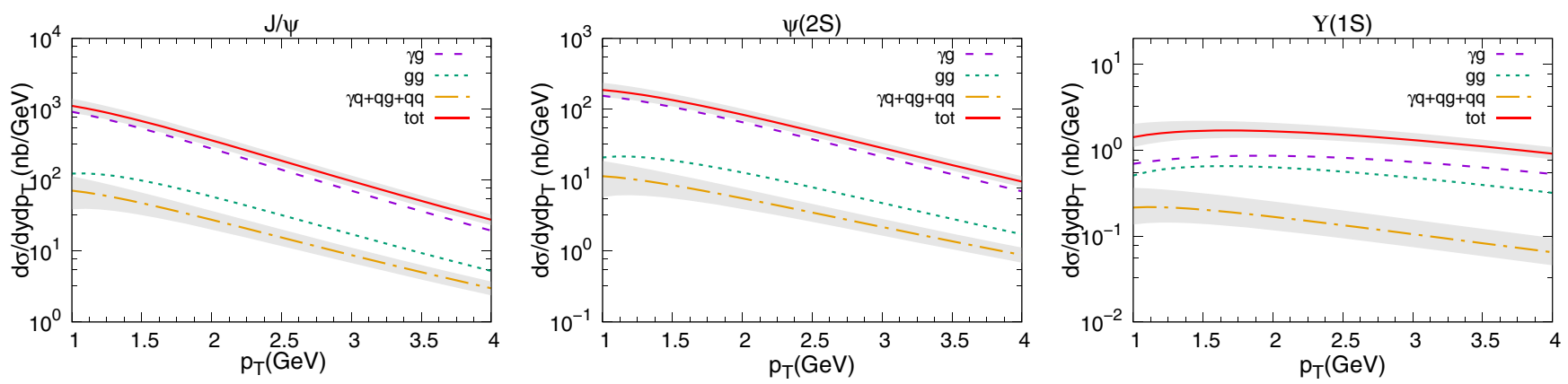

FIG. 8. Transverse momentum distributions for the inclusive diffractive $J / \Psi, \Psi(2 S)$, and $\Upsilon(1 S)$ photoproduction at central rapidities $(y=0)$ in $p \mathrm{~Pb}$ collisions at $\sqrt{s}=5.02 \mathrm{TeV}$. 

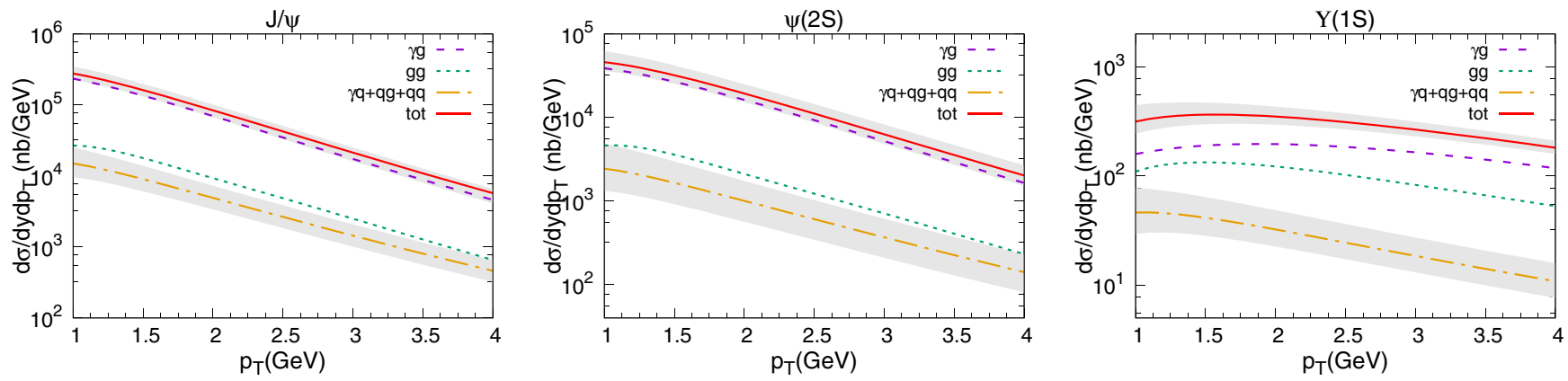

FIG. 9. Transverse momentum distributions for the inclusive diffractive $J / \Psi, \Psi(2 S)$, and $\Upsilon(1 S)$ photoproduction at central rapidities $(y=0)$ in $\mathrm{PbPb}$ collisions at $\sqrt{s}=5.02 \mathrm{TeV}$.

So, the contributions from quark-initiated channels are very close to the uncertainties of the total differential cross section. This indicates that the quark-initiated channels have significant modification to the quarkonium production. To improve the accuracy of the theoretical prediction, one should take into account the contributions from quark channels.

\section{SUMMARY}

We extend our previous work, which is based on the NRQCD factorization formalism, to include quark involved three subprocesses to study the heavy quarkonium production. We call this approach the quark improved NRQCD model. The quark improved NRQCD model is used to calculate the total cross section of the inclusive nondiffractive $J / \Psi$ production at HERA. By comparing our theoretical calculations of the $J / \Psi$ production with the experimental data, we find that the contributions from the three subprocesses mentioned above play a significant role in an accurate description of the inclusive nondiffractive heavy quarkonium production. Then we use the quark improved NRQCD model to study the inclusive diffractive heavy quarkonium photoproduction at LHC by combining with the resolved Pomeron model. We make the predictions of the inclusive diffractive rapidity and transverse momentum distributions of $J / \Psi, \Psi(2 S)$, and $\Upsilon(1 S)$ in $p p, p \mathrm{~Pb}$, and $\mathrm{PbPb}$ collisions. We estimate the percentage of the contributions from quark-initiated channels and the uncertainties of the total differential cross section, and find that the contributions from quark-initiated channels are very close to the uncertainties of the total differential cross section. These results show that the quark involved three subprocesses have significant modifications to the quarkonium production and may offer an insight to understand the underlying mechanism of heavy quarkonium production.

\section{ACKNOWLEDGMENTS}

This work is supported by the National Natural Science Foundation of China under Grants No. 11765005, No. 11305040, and No. 11947119; the Fund of Science and Technology Department of Guizhou Province under Grant No. [2019]5653; the Education Department of Guizhou Province under Grant No. KY[2021]131; and the China Postdoctoral Science Foundation under Grant No. 2018M633414.
[1] J. J. Aubert et al. (E598 Collaboration), Phys. Rev. Lett. 33, 1404 (1974).

[2] J. E. Augustin et al. (SLAC-SP-017 Collaboration), Phys. Rev. Lett. 33, 1406 (1974).

[3] J. Adam et al. (STAR Collaboration), Phys. Lett. B 797, 134917 (2019).

[4] U. Acharya et al. (PHENIX Collaboration), Phys. Rev. C 102, 014902 (2020).

[5] S. Acharya et al. (ALICE Collaboration), Phys. Lett. B 806, 135486 (2020).

[6] A. M. Sirunyan et al. (CMS Collaboration), Phys. Lett. B 790, 270 (2019).

[7] M. Aaboud et al. (ATLAS Collaboration), Eur. Phys. J. C 78, 171 (2018).

[8] R. Aaij et al. (LHCb Collaboration), J. High Energy Phys. 11 (2018) 194

[9] E. L. Berger and D. Jones, Phys. Rev. D 23, 1521 (1981).

[10] R. Baier and R. Ruckl, Phys. Lett. 102, 364 (1981).

[11] R. Baier and R. Ruckl, Z. Phys. C 19, 251 (1983).
[12] M. B. Einhorn and S. D. Ellis, Phys. Rev. D 12, 2007 (1975).

[13] C. H. Chang, Nucl. Phys. B 172, 425 (1980).

[14] C. E. Carlson and R. Suaya, Phys. Rev. D 14, 3115 (1976).

[15] H. Kowalski, L. Motyka, and G. Watt, Phys. Rev. D 74, 074016 (2006).

[16] A. H. Rezaeian and I. Schmidt, Phys. Rev. D 88, 074016 (2013).

[17] V. P. Goncalves, B. D. Moreira, and F. S. Navarra, Phys. Lett. B 742, 172 (2015).

[18] H. Fritsch, Phys. Lett. B 67, 217 (1977).

[19] F. Halzen, Phys. Lett. B 69, 105 (1997).

[20] F. Halzen and S. Matsuda, Phys. Rev. D 17, 1344 (1978).

[21] O. J. P. Eboli, E. M. Gregores, and F. Halzen, Phys. Rev. D 67, 054002 (2003).

[22] P. Hagler, R. Kirschner, A. Schafer, L. Szymanowski, and O. V. Teryaev, Phys. Rev. Lett. 86, 1446 (2001).

[23] F. Yuan and K. T. Chao, Phys. Rev. Lett. 87, 022002 (2001).

[24] S. P. Baranov, Phys. Rev. D 66, 114003 (2002).

[25] G. T. Bodwin, E. Braaten, and G. P. Lepage, Phys. Rev. D 51, 1125 (1995). 
[26] W. E. Caswell and G. P. Lepage, Phys. Lett. B 167, 437 (1986).

[27] G. P. Lepage, L. Magnea, C. Nakhleh, U. Magnea, and K. Hornbostel, Phys. Rev. D 46, 4052 (1992).

[28] G. A. Schuler, arXiv:hep-ph/9403387.

[29] F. Abe et al. (CDF Collaboration), Phys. Rev. Lett. 69, 3704 (1992).

[30] F. Abe et al. (CDF Collaboration), Phys. Rev. Lett. 79, 572 (1997).

[31] E. Braaten and T. C. Yuan, Phys. Rev. Lett. 71, 1673 (1993).

[32] E. Braaten and S. Fleming, Phys. Rev. Lett. 74, 3327 (1995).

[33] M. Cacciari, M. Greco, M. L. Mangano, and A. Petrelli, Phys. Lett. B 356, 553 (1995).

[34] M. Kramer, Prog. Part. Nucl. Phys. 47, 141 (2001).

[35] G. Baur, K. Hencken, D. Trautmann, S. Sadovsky, and Y. Kharlov, Phys. Rep. 364, 359 (2001).

[36] P. Ko, J. Lee, and H. S. Song, Phys. Rev. D 54, 4312 (1996).

[37] M. Cacciari and M. Kramer, Phys. Rev. Lett. 76, 4128 (1996).

[38] B. A. Kniehl and G. Kramer, Eur. Phys. J. C 6, 493 (1999).

[39] V. P. Goncalves and M. M. Machado, Eur. Phys. J. A 50, 72 (2014).

[40] V. P. Goncalves, L. S. Martins, and B. D. Moreira, Phys. Rev. D 96, 074029 (2017).
[41] V. P. Goncalves and B. D. Moreira, Phys. Rev. D 97, 094009 (2018).

[42] Y. Yang, S. H. Cai, Y. B. Cai, and W. C. Xiang, Nucl. Phys. A 990, 17 (2019).

[43] G. Ingelman and P. E. Schlein, Phys. Lett. B 152, 256 (1985).

[44] A. Aktas et al. (H1 Collaboration), Eur. Phys. J. C 48, 715 (2006).

[45] V. Guzey and M. Klasen, J. High Energy Phys. 04 (2016) 158.

[46] M. Gluck, E. Reya, and I. Schienbein, Phys. Rev. D 60, 054019 (1999).

[47] M. Drees and D. Zeppenfeld, Phys. Rev. D 39, 2536 (1989).

[48] E. Papageorgiu, Phys. Lett. B 250, 155 (1990).

[49] F. D. Aaron et al. (H1 Collaboration), Eur. Phys. J. C 68, 401 (2010).

[50] J. Pumplin, D. R. Stump, J. Huston, H. L. Lai, P. Nadolsky, and W. K. Tung, J. High Energy Phys. 07 (2002) 012.

[51] M. Klasen, B. A. Kniehl, L. N. Mihaila, and M. Steinhauser, Phys. Rev. D 68, 034017 (2003).

[52] G. M. Yu, Y. B. Cai, Y. D. Li, and J. S. Wang, Phys. Rev. C 95, 014905 (2017).

Correction: The omission of a "Corresponding author" label for the second author has been fixed. 